ARTICLE HISTORY: July: 19, 2021 Accepted: September 12, 2021 Published: September 19, 2021

РАСЧЕТ ПОТОКОВ В УСКОРИТЕЛЕ ЭЛЕМЕНТАРНЫХ ЧАСТИЦ

Якубовский Евгений Георгиевич

пенсионер

\title{
CALCULATION OF FLOWS IN AN ACCELERATOR OF ELEMENTARY PARTICLES
}

\author{
Yakubovsky Evgeny Georgievich \\ pensioner
}

\begin{abstract}
Аннотация. Уравнение Шредингера и Клейна-Гордона имеют конечное решение для скорости с помощью уравнения Навье-Стокса. Но уравнение Дирака не поддавалось решению с помощью конечной формулы для произвольного векторного и скалярного потенциала. Наконец сработал переход от производной функции, к производной от логарифма функции. Далее удалось решить линейное уравнение относительно производной от логарифма функции, которое возможно проинтегрировать. Причем оказалось, что возможно описать множество частиц. На ускорителях описывают траектории частиц с ошибкой, т.е. комплексные траектории. В данной статье поставлена задача рассчитать ускоритель в комплексной плоскости, где мнимая часть является ошибкой среднего - действительной части.

Abstract. The Schrödinger and Klein-Gordon equations have a finite velocity solution using the Navier-Stokes equation. But the Dirac equation did not lend itself to solving with the help of a finite formula for an arbitrary vector and scalar potential. Finally, the transition from the derivative of the function to the derivative of the logarithm of the function worked. Then we managed to solve a linear equation with respect to the derivative of the logarithm of the function, which can be integrated. Moreover, it turned out that it is possible to describe many particles. At accelerators, the trajectories of particles with an error are described, i.e. complex trajectories. In this article, the task is to calculate the accelerator in the complex plane, where the imaginary part is the error of the mean - the real part.

Ключевые слова: комплексные траектории элементарных частиц, расчет потоков в ускорителе, решение уравнения Дирака

Keywords: complex trajectories of elementary particles, calculation of flows in an accelerator, solution of the Dirac equation
\end{abstract}

Рассмотрим уравнение Дирака с произвольным векторным и скалярным потенциалом для множества частиц

$$
\sum_{\mu, k} \quad\left[\hat{p}_{\mu}-\frac{e}{c}\left(A_{\mu}\left(x_{p}\right)+\sum_{q=1}^{N} \quad A_{\mu}\left(x_{p}-x_{q}\right)\right)\right] \gamma_{i k}^{\mu} \psi_{k}\left(x_{p}\right)=m c \psi_{i}\left(x_{p}\right)
$$

формулу к зависимости от логарифма волновой функции и приводим подобные члены, получим

$$
\sum_{k} \quad \psi_{k}\left(x_{p}\right)\left\{\sum_{\mu} \quad\left[\hat{p}_{\mu} \gamma_{i k}^{\mu} \ln \psi_{k}\left(x_{p}\right)-\frac{e}{c}\left(A_{\mu}\left(x_{p}\right)+\sum_{q=1}^{N} \quad A_{\mu}\left(x_{p}-x_{q}\right) \gamma_{i k}^{\mu}\right)\right]-m c \delta_{i k}\right\}=0 .
$$

Где используются четырех векторы для описания разных частиц. Потенциал $A_{\mu}\left(x_{p}\right)$ - это внешнее воздействие. Кроме того, учитывается парное взаимодействие между частицами. Для тождественного равенства нулю волновой функции она должна равняться нулю с точностью до множителя, поэтому коэффициент перед ней должен быть равен нулю.

В данной формуле индексы i,k фиксированы и матрицы Дирака действительные

$$
\sum_{\mu} \quad\left[\hat{p}_{\mu} \gamma_{i k}^{\mu} \ln \psi_{k}\left(x_{p}\right)-\frac{e}{c}\left(A_{\mu}\left(x_{p}\right)+\sum_{q=1}^{N} \quad A_{\mu}\left(x_{p}-x_{q}\right)\right) \gamma_{i k}^{\mu}\right]=m c \beta_{i k} .
$$

Умножаем на обратную матрицу при фиксированном $\mathrm{k} \sum_{\mu, i} \quad \gamma_{v k i}^{-1} p_{\mu} \gamma_{i k}^{\mu}=\sum_{\mu} \quad p_{\mu} \delta_{v}^{\mu}=p_{v} ; \sum_{i} \quad \gamma_{v k i}^{-1} \gamma_{i k}^{\mu}=\delta_{v}^{\mu}$ получаем единичную матрицу относительно верхнего и нижнего индекса, получим при фиксированных $v, k$. Если элемент матриц $\gamma_{i k}^{\mu} \mathrm{c}$ индексом $\mathrm{k}$ тождественно равен нулю, то умножение на обратную матрицу не производим и обратную матрицу с этим элементом не считаем. Просуммируем по индексу k. Получим произведение компонент спиноров после умножения на обратную матрицу

$$
\sum_{k=1}^{4} \quad \hat{p}_{v} \ln \psi_{k}\left(x_{1}, \ldots, x_{N}\right) / 4=p_{v}\left(x_{1}, \ldots, x_{N}\right)=\frac{e}{c}\left[A_{v}\left(x_{p}\right)+\sum_{q=1}^{Q} \quad A_{v}\left(x_{p}-x_{q}\right)\right]+\sum_{k, i=1}^{4} \quad m c \gamma_{v k i}^{-1} \beta_{i k} / 4 .
$$


Так как левая часть уравнения мнимая, потенциалы должны зависеть от комплексных координат. Найдем линии тока уравнения Дирака, при комплексных начальных условиях, и вычислим потенциалы вдоль этих линий. Но предварительно надо произвести расчет электрических и магнитных напряженностей, обеспечивающих поток частиц в ускорителе. Это надо делать в комплексной плоскости. Зная комплексные напряженности электрического и магнитного поля произведем квантовый расчет ускорителя, его комплексных траекторий. Рассчитаем также траектории в системе центра инерции. На основе рассчитанного потенциала взаимодействия определяем массу образовавшейся частицы в результате столкновения.

$$
\begin{gathered}
\frac{d A_{v p}}{d s}=e_{v n m} H_{n}\left(x_{Q}\right) u_{m} / 2=e_{v n m} H_{n}\left(x_{v p}\right) p_{m p}\left(x_{v p}\right) / 2 m c ; p=1, \ldots, P ; v=1, \ldots, 3 \\
\frac{d A_{0 p}}{d s}=\sum_{n=1}^{3} E_{n}\left(x_{v p}\right) p_{n p}\left(x_{v p}\right) / m c ; A_{v p}=A_{v p}\left(s, x_{Q}^{0}\right) \\
\frac{d x_{v p}}{d s}=p_{v p}\left(x_{Q}\right) / m, x_{v p}=x_{v p}\left(s, x_{Q}^{0}\right) ; p=1, \ldots, P ; v=0, \ldots, 3 \\
x_{Q}=\left(x_{01}, x_{11}, x_{21}, x_{31}, \ldots, x_{0 Q}, x_{1 Q}, x_{2 Q}, x_{3 Q}\right) \\
x_{Q}^{0}=\left(x_{01}^{0}, x_{11}^{0}, x_{21}^{0}, x_{31}^{0}, \ldots, x_{k Q}^{0}, x_{1 Q}^{0}, x_{2 Q}^{0}, x_{3 Q}^{0}\right) .(1)
\end{gathered}
$$

Эти дифференциальные уравнения запишутся в комплексном пространстве, где мнимая часть удовлетворяет соотношению неопределенности и начальные условия комплексные. Действительное решение следует из условия непрерывности мнимой части решения. При этом другой соответствующий параметр комплексный и имеет бесконечную мнимую часть.

$$
\operatorname{Im} x_{v p} \sim s^{\alpha} ; \alpha<1 ; \frac{d \operatorname{Im} x_{v p}}{d s}=\operatorname{Im} p_{v p}\left(x_{Q}\right) / m=\lim _{s \rightarrow 0} \alpha s^{\alpha-1}=\operatorname{Im} \infty ; .
$$

Данные оценки следуют из свойств собственных значений. В случае невыполнения данной оценки мнимой части координаты, мнимая часть импульса является конечной и соотношение неопределенности не работает, дисперсия импульса существует. Существует две ветви квантовой механики, с действительным и комплексным пространством. Между ними граница, как между действительным ламинарным режимом и комплексным турбулентным режимом. Но нет критического числа Рейнольдса. Операторы самосопряженные для действительного пространства и общего вида для комплексного пространства. В действительной квантовой механике делаются попытки использовать для описания энергии, импульса и времени мнимые величины, но это вынужденные, силовые усилия. Собственные значения самосопряженных операторов действительные. Комплексные величины не могут быть собственными значениями операторов, так как они содержат мнимую часть, физический смысл которой переменная величина. Комплексные решения - это функции, описывающие траектории частиц в комплексном пространстве, аналог турбулентных линий тока.

Счет будем вести в системе центра инерции. По значению интервала определим начальные условия. Причем это начальное условие является продолжением решения (1), т.е. получаем, что решению с нулевым импульсом (2), соответствует решение (1). Тогда получаем формулу

$$
p_{v}\left(s, x_{Q}^{0}\right)=\frac{e}{c}\left[A_{v}\left(x_{p}\right)+\sum_{q=1}^{N} \quad A_{v}\left(x_{p}-x_{q}\right)\right]+\sum_{k, i=1}^{4} \quad m c \gamma_{v k i}^{-1} \beta_{i k} / 4=0 ; v=0, \ldots, 3
$$

Определив координаты при некотором значении интервала, следующие координаты определим из дифференциального уравнения

$$
\frac{\partial A_{\mu p}\left(x_{p}\right)}{\partial x_{v p}} \frac{d x_{v p}}{d s}=-\frac{d \sum_{q=1}^{N} \quad A_{\mu p}\left(x_{p}-x_{q}\right)}{d s} .
$$

При столкновении правая часть дифференциального уравнения стремится к бесконечности, значит и скорость, и импульс стремится к бесконечности в одной точке. Это означает, что координата испытывает скачок. Докажем это по-другому. Третье дифференциальное уравнение (1) имеет особенность, бесконечность правой части в точке столкновения. А это означает нарушение непрерывности решения и скачок координаты см. [1], т.е. скачкообразное изменение потенциала, или изменение массы рассеянной частицы.

Начальные условия выберем с поперечным радиусом удовлетворяющем гауссовой поверхности. Т.е. со сгущением к центру потока. Дисперсию гауссовой кривой надо задавать, ее можно оценить из эксперимента. Мнимую часть начальных условий выберем средней для расстояния между соседними точками.

Значение энергии определится из знания скалярного потенциала электрического поля $e\left[A_{0}\left(x_{p}\right)+\right.$ $\left.\sum_{q=1}^{N} \quad A_{0}\left(x_{p}-x_{q}\right)\right]=-m c^{2} \sum_{k, i=1}^{4} \quad \gamma_{0 k i}^{-1} \beta_{i k}$. 
При этом энергия определится однозначно в системе центра инерции $H=m c^{2}\left(1+\sum_{k, i=1}^{4} \quad \gamma_{0 k i}^{-1} \beta_{i k} / 4\right)$.

Получается, что каждая частица находится в системе центра инерции. Частицы либо столкнутся, с совпадением действительной части координаты, либо разлетятся. При этом мнимая часть координаты отличается. Их энергия либо останется неизменной, либо образуются несколько новых частиц, масса которых определится из уравнения. Свойства предела передаются от доминирующего потенциала.

$$
m_{x v}=\frac{-e\left[A_{v}\left(x_{p}\right)+\sum_{q=1}^{N} \quad A_{\nu}\left(x_{p}-x_{q}\right)\right]}{c^{2} \sum_{k, i=1}^{4} \quad \gamma_{v i k}^{-1} \beta_{i k}},(4)
$$

Для остальных частиц масса не изменится. Образоваться новая частица может за счет совпадения действительных координат, при малой разнице мнимых координат, тогда потенциал возрастет, и новая частица образуется, сечение реакции велико. Параллельно для получения скачка координаты, надо считать знаменатель только с действительной частью и при нуле знаменателя произойдет скачок. Но при решении уравнения (3) надо воспользоваться неявной схемой решения.

$$
\operatorname{Re} x_{v p}=\operatorname{Re}\left\{x_{v p 0}-\left[\frac{\partial A_{\mu p}\left(x_{p}\right)}{\partial x_{v p}}\right]^{-1} \frac{d \sum_{q=1}^{N} A_{\mu p}\left(\operatorname{Re} x_{p}-\operatorname{Re} x_{q 0}\right)}{d s} \Delta s\right\}
$$

Тогда бесконечность знаменателя не произойдет и определится выход из системы центра инерции при минимальной разности сталкивающихся координат. Для возврата в систему центра инерции надо изменить массу. Импульс частицы состоит из двух частей, материальной и полевой. В сумме они равны нулю. Эта формула в случае образования двух частиц, каждая со своей массой. В случае образования трех частиц одна из них распадется в перпендикулярном направлении на суммарный нулевой импульс.

Сечение рассеяния определится из формулы

$$
\begin{gathered}
\sigma=\frac{\pi \hbar^{2}}{p^{2}} \frac{\left|\operatorname{Re} x_{p}\left(t_{1}+\frac{\hbar}{\Gamma}\right)-\operatorname{Re} x_{q}\left(t_{1}+\frac{\hbar}{\Gamma}\right)\right|^{2}}{\left|\operatorname{Im} x_{p}\left(t_{1}\right)-\operatorname{Im} y_{q}\left(t_{1}\right)\right|^{2}+\left|\operatorname{Re} x_{p}\left(t_{1}+\frac{\hbar}{\Gamma}\right)-\operatorname{Re} x_{q}\left(t_{1}+\frac{\hbar}{\Gamma}\right)\right|^{2}} ; \sigma \frac{\pi \hbar^{2}}{p^{2}} \max ^{\operatorname{Rax}} \\
\operatorname{Rex}_{p}\left(t_{1}\right)=\operatorname{Re} x_{q}\left(t_{1}\right) ; x_{p}(t) \neq x_{q}(t) ; p=\sqrt{\sum_{v=1}^{3}\left(m c \sum_{k=1}^{4} \gamma_{v k k}^{-1} / 4\right)^{2} ; \Gamma=\frac{m_{e} c^{2}}{137^{2}}+\Gamma_{1}+\Gamma_{2}}
\end{gathered}
$$

В случае бесконечного времени жизни получаем максимальную добавку к времени столкновения. К сожалению лобовой удар невозможен из-за конечного размера частицы. Частицы не являются точечными, имеют конечные размеры и это надо учитывать при вычислении сечения реакции. При лобовом столкновении отличаются действительные и мнимые координаты из-за конечных размеров частицы и сечение рассеяния уменьшается. Поэтому в знаменателе и в числителе надо вводить не нулевую разность действительных координат.

Выводы

Сначала я просто использовал формулу связи между квантовой механикой и уравнением Навье-Стокса, $V_{k}=$ $-i \frac{\hbar}{m} \frac{\partial \ln \psi}{\partial x_{k}}$, где используется скорость из уравнения Навье-Стокса, и волновая функция квантовой механики. Потом построил комплексные скорости квантовой механики, определив линии тока. Аналогия с турбулентным потоком прояснилась. Турбулентный поток тоже описывается комплексным скоростями. Действительная часть комплексной скорости - это среднее, а мнимая часть - это среднеквадратичное отклонение. И то, и другое есть в квантовой механике и турбулентном потоке. Отличие в деталях, квантовая механика использует электромагнитную энергию, а турбулентный поток - гидродинамическую, звуковую. Кинематическая вязкость квантовой механики мнимая и равна $i \frac{\hbar}{2 m}$, кинематическая вязкость турбулентного потока действительная. Но имеется квантовая механика в действительном пространстве. Аналогии в классической физике не имеет. Ламинарное решение тоже действительное, но имеются линии тока, чего нет в квантовой механике в действительном пространстве. Кроме того, ламинарное решение не имеет собственных значений. Но граница между комплексным и действительным решением в квантовой механике и гидродинамике одинаково существует.

И та, и другая теория подчиняется преобразованию Лоренца, но квантовая механика со скоростью света, а гидродинамика со скоростью звука. Имеется интервал, электромагнитных и звуковых волн и вывод уравнений Лоренца аналогичен в электромагнитных и звуковых волнах. Скорость возмущения равна групповой скорости для звука, а значит групповой скорости для электромагнитной волны. По поводу преобразования Лоренца есть интернет книга см. [2]. Обе скорости не зависят от скорости центра инерции бесконечной среды. Но имеется отличие, релятивистский знаменатель со скоростью звука относится к присоединенной массе, а со скоростью света к инерционной массе. Формула сложения скоростей справедлива для среды в случае гидродинамики, и для 
инерционной массы в случае электромагнитной скорости света. Групповая скорость для элементарных частиц совпадает со скоростью света в вакууме.

\section{Литература}

[1] Якубовский Е.Г. Скачки решения системы обыкновенных нелинейных дифференциальных уравнений «Энциклопедический фонд России», 2019, 9 cтp. http://www.russika.ru/sa.php?s=1237

[2] Якубовский Е.Г. По поводу преобразований Лоренца «Энциклопедический фонд России», 2019, 115 стр. http://www.russika.ru/userfiles/390_1620069631.pdf

[1] Yakubovskiy E.G. Jumps of the solution of a system of ordinary nonlinear differential equations "Encyclopedic Foundation of Russia", 2019, 9 pages. http://www.russika.ru/sa.php?s=1237

[2] Yakubovskiy E.G. Concerning the Lorentz transformations "Encyclopedic Fund of Russia", 2019, 115 pages. http://www.russika.ru/userfiles/390_1620069631.pdf 